\title{
The syllable-length effect in number processing is task-dependent
}

\author{
INGRID GIELEN, MARC BRYSBAERT, and ANNIE DHONDT \\ University of Leuven, Leuven, Belgium
}

\begin{abstract}
Two experiments were run in order to investigate the relationship between syllable length of number names and eye-fixation durations during silent reading of one- and two-digit numbers. In Experiment 1, subjects had to read a series of three numbers and recall them orally; in Experiment 2 , subjects had to indicate manually whether the value of the middle number was between the values of the outer numbers. The effect of syllable length was controlled for possible confounding effects of number frequency and number magnitude. Findings indicated that fixation duration depended on syllable length of number names in the first task, but not in the second task. The results call into question the claim that phonological encoding is imperative in visual processing; phonological encoding was used only when the numbers had to be recalled, and not when they were coded for computational purposes.
\end{abstract}

Pynte (1974) showed that the relationship between syllable length of number names and response latency previously found for number naming (Eriksen, Pollack, \& Montague, 1970; Klapp, 1971, 1974) also existed in silent number reading. Two series of three two-digit numbers were each presented for $2 \mathrm{sec}$. Subjects had to read the numbers silently and to report them aloud as soon as the stimulus display disappeared. Eye movements were tracked while the subjects were reading. Pynte (1974) found that gaze duration on the center number increased as the syllable length of the number name increased, although pronunciation was not required during processing. This led Pynte to the conclusion "that a mechanism of readiness for pronunciation co-occurs with visual processing" (p. 110).

Pynte's (1974) finding is regularly cited, along with other studies, as evidence that phonological encoding is an essential element in visual processing (see, e.g., Hung \& Tzeng, 1981; Rayner \& Pollatsek, 1989, p. 214; Rossi, 1985). However, before Pynte's (1974) results can be used as unequivocal support for phonological mediation in reading, alternative interpretations must be ruled out. Pynte used only two times two critical stimuli: "30" (trente, one syllable) versus " 90 " (quatre-vingt-dix, four syllables), and " 28 " (vingt-huit, two syllables) versus " 82 "' (quatre-vingt-deux, four syllables). This leaves open the possibility that the findings were due to factors other than syllable length of the stimulus name. First, Pynte did not consider the frequency of occurrence of his stimuli, so that his results may have been an artifact of the large num-

The research was partially supported by a grant from the G.O.A. (Geconcerteerde Onderzoeksacties) of the Belgian Government, Convention Number 87/93-111. Correspondence should be addressed to Marc Brysbaert, Laboratory of Experimental Psychology, University of Leuven, Tiensestraat 102, B-3000 Leuven, Belgium (e-mail: fpaas02@blekul11). bers' having lower frequencies than the low numbers. Second, it is not unreasonable to assume that large numbers are more difficult to process than small numbers, so that Pynte's syllable effect might also have been due to differences in number magnitude. Finally, it can be asked whether Pynte really did design a task "without pronunciation." Though naming was not required during reading, the subsequent oral report of numbers may have entailed subvocalization as part of response preparation.

To address the three criticisms above, we ran two replications of Pynte (1974), in which all of the important factors were controlled: In Experiment 1, a number recall task similar to Pynte's was used. In Experiment 2, subjects had to compare numbers without vocal report. The weight of each critical factor (syllable length, number frequency, and number magnitude) was assessed with multiple regression analyses on the eye-fixation data.

\section{EXPERIMENT 1}

To investigate whether Pynte's (1974) syllable-length effect remained valid if number frequency and number magnitude were partialed out, the paradigm was repeated for all numbers smaller than 100 , instead of the original pairs $30-90$ and $28-82$. Because there were no objective measurements of number frequency available, subjective estimates were used (see Method).

\section{Method}

Subjects. Eleven native Dutch-speaking undergraduate psychology students from the University of Leuven participated in the experiment. None of the subjects wore glasses or lenses. All were unaware of the purpose of the study. The data of 2 additional subjects were excluded on line because of unsatisfactory eye-movement registrations (see the section on dependent variables).

Stimull. The stimulus display of each trial consisted of three numbers, presented on the left, center, and right of a CRT screen (see Figure 1). One hundred trials of three random numbers were 


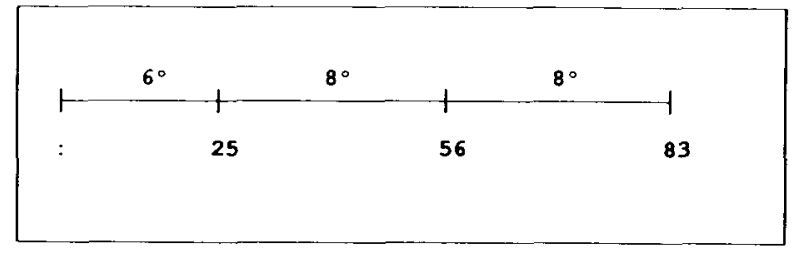

Figure 1. Stimulus display.

presented to all subjects. Numbers and trials were randomized, with the following constraints: (1) All numbers from 0 to 99 appeared once as the left, middle, and right numbers, and (2) all three numbers in each display were either single-digit or double-digit numbers. Each subject received a different set of stimuli (for more information, see Brysbaert, 1991). The subjects sat $70 \mathrm{~cm}$ in front of an ETAP screen, a large CRT screen that subtends $36 \mathrm{~cm}$ horizontally (i.e., $27^{\circ}$ at a distance of $70 \mathrm{~cm}$ ) and $26 \mathrm{~cm}$ vertically. It made use of the fast-decaying P4 phosphor. The three numbers of a trial were separated from one another by a visual angle of $8^{\circ}$, in order to exclude parafoveal preprocessing. Digits were $.57^{\circ}$ high and $.48^{\circ}$ wide.

Task. The subjects were instructed to look at the fixation point (colon) on the left side of the screen (see Figure 1) until a trial was presented. Adequate fixation was ensured with the eye-movement system (see below). As soon as the numbers appeared, the subjects had to read them silently from left to right. The stimuli were presented for $2 \mathrm{sec}$, after which the subjects repeated them aloud. The experimenter recorded the subjects' accuracy on the center number. Trials in which eye-movement registration was deficient were repeated at a random place later in the series. This occurred on 14 out of a total of 1,100 trials.

Independent variables. Because all numbers from 0 to 99 were used, number magnitude ranged from 0 to 99 and syllable length of number names (in Dutch) from 1 to 6 . Number frequency was assessed with a questionnaire. Twenty students, from the same population as the subjects in the experiment, rated the numbers on a scale ranging from 1 (extremely rare) to 6 (extremely frequent) with respect to frequency in everyday life. Afterward, the answers were linearly rescaled (by subtracting 1 and multiplying the result by 100) in order to get a frequency range between 0 and 500 . In this way, integer numbers were obtained without their being rounded off. Previous research with words had indicated that such subjective estimates of frequency are both reliable (Galbraith \& Underwood, 1973; Shapiro, 1969) and highly correlated with objective and tabulated measurements (Gernsbacher, 1984). The values of the three independent variables for all numbers are listed in the Appendix.

Dependent variables. Eye movements were registered with Debic-80 equipment on the basis of pupil-center corneal reflection (Young \& Sheena, 1975), with a sampling rate of $50 \mathrm{~Hz}$. The system was accurate to $0.5^{\circ}$ and was interfaced with a PDP-11/40 computer that kept a complete record of the $x$ and $y$ coordinates of the subject's point of regard. During the measurement, the experimenter could follow the point of regard on a monitor, in order to check whether the subjects looked at the fixation point before the stimuli appeared and whether the eye-movement registration was satisfactory. Raw data were transformed to fixations by means of a data reduction program (based on Kliegl \& Olson, 1981). A fixation was defined as a sequence of at least five consecutive measurements (i.e., $100 \mathrm{msec}$ ) whose $x$ and $y$ coordinates did not deviate more than $1^{\circ}$ horizontally and $0.75^{\circ}$ vertically. Because estimates of fixation durations based on reduction programs outlined above are sensitive to missing values, an analysis of the percentage of missing values was performed. The percentage amounted to $1.5 \%$ and was not correlated with any of the independent variables (correlations were smaller than +.10 and -.10 ).
Only fixations on the center number were analyzed. A distinction was made between first-fixation duration (FFD) and first-gaze duration (FGD). FFD is the duration of the very first fixation on the center number. FGD is the sum of FFD and all consecutive fixations the first time the center number is looked at (i.e., without regressive gazes). The distinction between FFD and FGD is commonly made in eye-movement research (De Graef, Christiaens, \& d'Ydewalle, 1990; Inhoff, 1984; Rayner \& Pollatsek, 1987), because it is believed that FFD reflects initial processing, whereas FGD also includes relatively late cognitive processing. Thus the distinction can give some information about whether phonological information is accessed during encoding (FFD) and/or during further processing (FGD).

Procedure. Each subject was tested individually, after an explanation of the experimental setting and task. The subject sat in an adjustable chair $70 \mathrm{~cm}$ in front of a CRT screen. The chair could be adjusted for height and position of the headrest. In addition, a chinrest was used to prevent small head movements. Before the 100 test trials were administered, the eye-movement registration system was calibrated and 10 practice trials with letters instead of numbers were presented to ensure that the subject understood the instructions correctly. After the test, subjects received feedback about their performance. The whole experimental session took about $35 \mathrm{~min}$.

\section{Results}

The three predictor variables-syllable length, number frequency, and number magnitude-were highly intercorrelated. Pearson correlation coefficients amounted to $-.753(n=100, p<.01)$ between syllable length and number frequency; $.718(n=100, p<.01)$ between syllable length and number magnitude; and $-.621(n=100$, $p<.01$ ) between number frequency and number magnitude. The substantial negative correlation between syllable length and number frequency is interesting because it is in line with word findings (cf. the so-called Zipf law; Zipf, 1935, 1949). At the same time, the high correlations between the predictor variables support our criticisms of Pynte's (1974) results.

The subjects' error rate was $4.7 \%$. Analysis of the eyefixation data revealed no significant difference between correct and incorrect trials. Therefore, the remaining analyses are based on all trials. ${ }^{1}$ In addition, 13 missing cells (i.e., 1.2\%) had to be estimated with the procedure from Yates (1933), which enables one to calculate missing observations from the sum of the subject's other observations and the sum of the remaining observations for the stimulus in question (see also Kirk, 1982, pp. 268-270). Mean FFD of the results thus obtained amounted to 266 msec; mean FGD was 404 msec. Mean FFD and FGD per number are presented in the Appendix.

Correlations between the predictor variables and the eye-fixation data are listed in Table $1 .^{2}$ The first line for each dependent variable, FFD and FGD, gives the raw Pearson correlation coefficients; the second line shows the correlations when the effects of the two other variables are partialed out; the third line, finally, lists the results of a model that will be explained below. Syllable length correlated significantly with both FFD and FGD, even when the effects of number frequency and number magnitude were partialed out. This means that Pynte (1974) 
Table 1

Raw Correlations, Highest Order Partial Correlations, and Partial Correlations According to the Model for First-Fixation Duration and First-Gaze Duration in the Number-Recall Task (Experiment 1)

\begin{tabular}{|c|c|c|c|}
\hline & $\begin{array}{c}\text { Syllable } \\
\text { Length }\end{array}$ & $\begin{array}{l}\text { Number } \\
\text { Frequency }\end{array}$ & $\begin{array}{c}\text { Number } \\
\text { Magnitude }\end{array}$ \\
\hline \multicolumn{4}{|c|}{ First-Fixation Duration } \\
\hline $\begin{array}{l}\text { Raw correlation } \\
\text { Highest order partial correlation } \\
\text { Partial correlations model } \dagger\end{array}$ & $\begin{array}{l}.401^{*} \\
.270^{*} \\
.401^{*}\end{array}$ & $\begin{array}{l}-.366^{*} \\
-.140 \\
-.106\end{array}$ & $\begin{array}{r}.181 \\
-.191 \\
-.191\end{array}$ \\
\hline \multicolumn{4}{|c|}{ Final model: $F F D=217.24+13.11 \mathrm{Syl}$} \\
\hline \multicolumn{4}{|c|}{ First-Gaze Duration } \\
\hline $\begin{array}{l}\text { Raw correlation } \\
\text { Highest order partial correlation } \\
\text { Partial correlations model } \dagger\end{array}$ & $\begin{array}{l}.678^{*} \\
.326^{*} \\
.678^{*}\end{array}$ & $\begin{array}{l}-.667^{*} \\
-.322^{*} \\
-.323^{*}\end{array}$ & $\begin{array}{r}.506^{*} \\
-.020 \\
-.020\end{array}$ \\
\hline \multicolumn{4}{|c|}{ Final model: FGD $=399.62+25.30$ Syl -0.36 Freq } \\
\hline
\end{tabular}

has been successfully replicated. Number frequency was also correlated with FFD and FGD, and number magnitude was correlated with FGD (see Table 1); but only the correlation between number frequency and FGD remained significant when the effect of the other variables was partialed out.

Raw correlations inflate the importance of predictor variables if these variables are interrelated. Highest order partial correlations, on the other hand, underestimate the true weights, because the effect of each variable is partialed out for possible effects of all other variables. Therefore, a model was developed in which the variables were entered successively. In this way, correlations were only partialed out for effects of previously entered factors. To develop such a model, it is customary to enter the variables with the largest correlations first. However, because the differences between the correlations for syllable length and number frequency were only marginal, it was not evident which of the two predictors dominated.

Therefore, we ran an analysis of variance (ANOVA) on a subset of 16 two-digit numbers for which the predictor variables were independent. A $2 \times 2 \times 2$ design was obtained by taking high and low values for each variable. Each cell contained two numbers with an average value 1.5 SDs above or below the mean. These numbers are indicated in the Appendix with an asterisk. No effect reached significance in the analysis of FFD. In the analysis of FGD, however, there was a clear effect of both syllable length [short length, $335 \mathrm{msec}$; long length, $419 \mathrm{msec}$; $F(1,10)=10.68, p<.01]$ and number frequency [low frequency, $391 \mathrm{msec}$; high frequency, $363 \mathrm{msec} ; F(1,10)$ $=5.17, p<.05]$. The effect of syllable length was more pronounced both in $F$ values and in variance components. Because the same tendency was present for FFD, it was concluded that the slightly larger correlation between syllable length and the eye-fixation data (see Table 1) indeed indicated a predominance of syllable length over number frequency.
On the basis of the results of the 16 two-digit numbers, partial correlations for all 100 stimuli were calculated according to the following model: 1 = syllable length, 2 = number frequency, and $3=$ number magnitude. The partial correlations according to this model are presented in Table 1, together with the final regression models for FFD and FGD. For FFD, only syllable length remained significant. For FGD, both syllable length and number frequency attained significance.

\section{Discussion}

In the introduction, it was argued that the effect of syllable length on number processing obtained by Pynte (1974) might have been an artifact of number frequency or number magnitude. Analysis of the numbers ranging from 0 to 99 indicated that these three variables are indeed highly correlated (correlations of .60 and more). The correlation between syllable length and number magnitude is inherent in the way Dutch (French, English) number names are constructed. The correlation between syllable length and number frequency is in line with word findings, where it has been shown that high-frequency words tend to be shorter than low-frequency words (see, e.g., Zipf, 1935, 1949). Therefore, Pynte's (1974) stimuli differed not only in number of syllables, but also with respect to number magnitude and probably number frequency. ${ }^{3}$ (See the Appendix.)

In order to control for confounding effects of number frequency and number magnitude, multiple regression analyses on eye-fixation data for all numbers smaller than 100 were run. This was preferred to the analysis of an artificial subset of numbers that orthogonally varied on each of the variables of interest (though see the Results section). The regressions indicated not only that syllable length explained most of the variance (16\% for FFD, $46 \%$ for FGD) but also that the effect remained significant when the impact of number frequency and magnitude was partialed out. This was true for both FFD and FGD, which 
seems to indicate that the phonological information is used from the first processing stages on. In addition, FGD was significantly influenced by the subjective frequency of the numbers. The effect of syllable length amounted to $13 \mathrm{msec}$ per syllable for FFD and 25 msec for FGD. Mean FFD (266 msec) and FGD (404 msec) are comparable to values obtained in word reading (see, e.g., Inhoff, 1984; Rayner \& Pollatsek, 1989, p. 115).

The successful replication of Pynte (1974) indicates, after elimination of potential artifacts, that phonological encoding is indeed part of visual processing in this task, as argued by, for instance, Rayner and Pollatsek (1989, p. 214), and Rossi (1985). The finding is the more compelling because vocalization was not required during the encoding stage in which eye movements were measured. However, it can be asked to what extent the results above relate to visual processing of numbers in general. First, the phonological encoding may have been provoked by the subsequent vocal recall of the numbers. Second, numbers were treated as character strings that merely had to be remembered and that in principle could have been replaced by any kind of symbols with a name. The more specific computational use of numbers (i.e., addition, multiplication) was not addressed. Both criticisms were dealt with in the next experiment.

\section{EXPERMMENT 2}

Experiment 2 tested whether the observed syllablelength effect in number processing would be present in a nonrecall task. In Experiment 1, vocalization was required for the output, and numbers constituted a nominal scale; that is, they were just labels that identified the elements of a population (Stevens, 1951). The normal computational use of numbers involves at least an ordinal scale and most often an interval or even a ratio scale. Therefore, Pynte's (1974) task was modified. Subjects again saw a series of three numbers ranging from 0 to 99 , but this time they had to indicate manually whether the value of the middle number was between the values of the outer numbers. If the syllable-length effect could be obtained for this task as well, the evidence that phonological encoding is a necessary component of visual number processing would become stronger.

\section{Method}

Subjects. Eleven new subjects from the same population as that used in Experiment 1 participated. None wore glasses or lenses. All were unaware of the purpose of the study. The data of 4 additional subjects were excluded because of unsatisfactory eye-movement registrations.

Stimuli. Again, 100 trials of three random numbers were presented to all subjects. During these trials, all numbers ranging from 0 to 99 were presented once in the middle position. In half of the trials, the value of the middle number was between the values of the two outer numbers, which were randomly selected. In the other half, the value of the middle number was above or below the values of the outer numbers. Single-digit numbers were always coupled with other single-digit numbers, two-digit numbers with two-digit numbers. Each middle number had a .5 probability of falling be- tween the other two numbers. This was not true for the numbers 0 and 10 , which constituted the lower limit, or for 9 and 99 , which constituted the upper limit; these values were always situated outside the range of the outer numbers. The stimuli were constructed in such a way that the processing of all three numbers was necessary for subjects to perform the task correctly. Each subject received a different stimulus constellation. Presentation occurred in the same way as it did in Experiment 1.

Task. The subjects were instructed to look at the fixation point on the left side of the screen until a stimulus was presented. Adequate fixation was ensured with the eye-movement system. As soon as the numbers appeared, the subjects had to read them silently from left to right. They had to press the right button if the value of the middle number was situated between the outer two, and the left button otherwise. The subjects were instructed to respond as soon as they knew the answer. The stimuli were presented until the subject reacted or for a maximal period of $2 \mathrm{sec}$. Trials in which eyemovement registration was deficient were repeated at a random place later in the series. This occurred on 11 out of a total of 1,100 trials.

Independent and dependent variables; Procedure. As in Experiment 1 , the independent variables were syllable length of the number names, number frequency, and number magnitude. Raw data were transformed to fixations with the programs used in Experiment 1 . The percentage of missing values amounted to $3.4 \%$ and was not correlated with any of the independent variables. FFD and FGD on the center number were analyzed. Reaction times were also collected, but they were not analyzed, because they were the result of many more factors than the one we were interested in (e.g., they involved identification and comparison of the three numbers, together with a correct manual response). The procedure was the same as it was in Experiment 1.

\section{Results}

The mean reaction time was $2.24 \mathrm{sec}$ for all 1,100 trials, and it ranged from 1.76 to $3.60 \mathrm{sec}$ for individual subjects. Error rate was $8.82 \%$. No significant differences in eye-fixation data were found between correct and incorrect trials. Therefore, the remaining analyses are based on all trials (see note 1). Twenty-one missing cells (i.e., $1.9 \%$ ) had to be estimated with the procedure from Yates (1933; see above). Mean FFD was 236 msec; mean FGD was $304 \mathrm{msec}$. FFD was not significantly shorter in Experiment 2 than it was in Experiment $1[F(1,20)=$ $3.44, p>.05]$; FGD, however, was $[F(1,20)=12.05$, $p<.01]$. Mean FFD and FGD per number are presented in the Appendix.

Pearson correlation coefficients between the predictor variables and the eye-fixation data for Experiment 2 are listed in Table 2. The outline of this table is the same as that of Table 1 . Only number magnitude was correlated significantly with FFD; the correlation between FFD and syllable length did not reach significance. For FGD, the correlations with number magnitude and with syllable length were significant (but see note 2). However, when the correlations were partialed out for the other effects, none of them remained significant.

As in Experiment 1, an ANOVA was run on the subset of 16 numbers for which the predictor variables were orthogonal, in order to have additional evidence of the relative importance of the three predictor variables (cf. the correlation between FGD and number magnitude is not much larger than the correlation between FGD and 
Table 2

Raw Correlations, Highest Order Partial Correlations, and Partial Correlations According to the Model for First-Fixation Duration and First-Gaze Duration in the Number-Comparison Task (Experiment 2)

\begin{tabular}{|c|c|c|c|}
\hline & $\begin{array}{l}\text { Syllable } \\
\text { Length }\end{array}$ & $\begin{array}{l}\text { Number } \\
\text { Frequency }\end{array}$ & $\begin{array}{c}\text { Number } \\
\text { Magnitude }\end{array}$ \\
\hline \multicolumn{4}{|c|}{ First-Fixation Duration } \\
\hline $\begin{array}{l}\text { Raw correlation } \\
\text { Highest order partial correlation } \\
\text { Partial correlations model* }\end{array}$ & $\begin{array}{l}.190 \\
.108 \\
.043\end{array}$ & $\begin{array}{r}-.077 \\
.127 \\
.127\end{array}$ & $\begin{array}{l}.224 t \\
.149 \\
.224 t\end{array}$ \\
\hline \multicolumn{4}{|c|}{ Final model: $F F D=223.14+0.25 \mathrm{Mag}$} \\
\hline \multicolumn{4}{|c|}{ First-Gaze Duration } \\
\hline $\begin{array}{l}\text { Raw correlation } \\
\text { Highest order partial correlation } \\
\text { Partial correlations model* }\end{array}$ & $\begin{array}{l}.231 \ddagger \\
.176 \\
.089\end{array}$ & $\begin{array}{r}-.072 \\
.182 \\
.182\end{array}$ & $\begin{array}{l}.238 \ddagger \\
.139 \\
.238 \ddagger\end{array}$ \\
\hline \multicolumn{4}{|c|}{ Final model: $F G D=285.87+0.36 \mathrm{Mag}$} \\
\hline
\end{tabular}

syllable length). No effect reached significance in the ANOVA on FFD. In the analysis of FGD, there was an effect of both number magnitude [small numbers, $291 \mathrm{msec}$; large numbers, $320 \mathrm{msec} ; F(1,10)=6.46, p<.05]$ and number frequency [low frequency, $289 \mathrm{msec}$; high frequency, $322 \mathrm{msec} ; F(1,10)=6.34, p<.05]$. No effect was present for syllable length $[F(1,10)=1.49, p>.10]$. The variance component of number magnitude was also larger than the variance component of syllable length in the analysis of FFD.

Therefore, the order in which the variables were entered into the model for the regression analysis on all 100 numbers was: 1 = number magnitude, 2 = syllable length, and 3 = number frequency. Syllable length was entered before number frequency because of its larger correlation with the eye-fixation data in the regression analyses, and because the frequency effect in the ANOVA was not in line with other frequency effects reported in the literature. That is, FGD was longer for high-frequency numbers than for low-frequency numbers (see above). The partial correlations according to the model outlined above are presented in Table 2, together with the final models for FFD and FGD. In both models, only the effect of number magnitude remains significant. The effect of syllable length is negligible (less than $1 \%$ of the variance explained).

It might be wondered whether the shorter FGD in Experiment 2 as opposed to Experiment 1 (see above) was compensated for by more regressive gazes, so that the syllable-length effect has been underestimated by FGD. To check this possibility, correlations between the predictor variables and regressive gaze durations (RGD) were calculated. RGDs are the sum of all fixations after FGD. They could be due either to a refixation of the middle number after a regression to the first number, or to a regression from the third number. Although RGD was longer in Experiment $2(M=260 \mathrm{msec})$ than it was in Experiment 1 $(117 \mathrm{msec})$, no correlation between RGD and the predictor variables was larger than $.10(n=100, p>.20)$. For syllable length, the correlation was even negative $(r=-.02)$.

Another criticism might be that Experiment 2 did not provide the opportunity for the syllable-length effect to emerge that was provided by Experiment 1.4 It could be argued that subjects did not have to process the whole number in order to complete the task successfully, but that most of the time the first digit of the numbers was sufficient for the subjects to determine whether the middle number was between the other two in magnitude. For instance, a subject might decide whether the value of the middle number was situated between the outer two in a series " 123486 " simply by looking at the first digits " 13 8." This, of course, would eliminate any syllablelength effect in the regression analyses, because the range of syllable lengths would be reduced from six to two, with a clear predominance of monosyllabic "numbers."

To check the plausibility of this alternative explanation, a new analysis of the eye-movement data was performed. If subjects indeed used the strategy of only looking at the first digit, a rather clear prediction follows-namely, that a regressive eye movement will be made if there is a tie between first digits (e.g., when the series " 212395 " is presented). Therefore, the mean number of saccades for different stimulus configurations was calculated (see Table 3).

There are nine possible saccades between the three numbers of a stimulus display; these saccades can be divided into refixations (i.e., a new fixation on the same number), progressions (i.e., from a number to the left to a number to the right), and regressions (from a number to the right to a number to the left). There were on the average 3.71 saccades (see the second to last line of Table 3). Two of these saccades were, not unexpectedly, progressions from the first to the second number, and from the second to the third number. Regressions were also quite common, and accounted for a mean of 1.31 saccades. 
Table 3

Mean Number of Saccades between the Three Numbers (Divided into Refixations, Progressions, and Regressions), Sum of Saccades, Number of Observations, Percentage of Correct Responses, and Mean Correct Response Times (RT) to Different Stimulus Configurations in the Number-Comparison Task (Experiment 2)

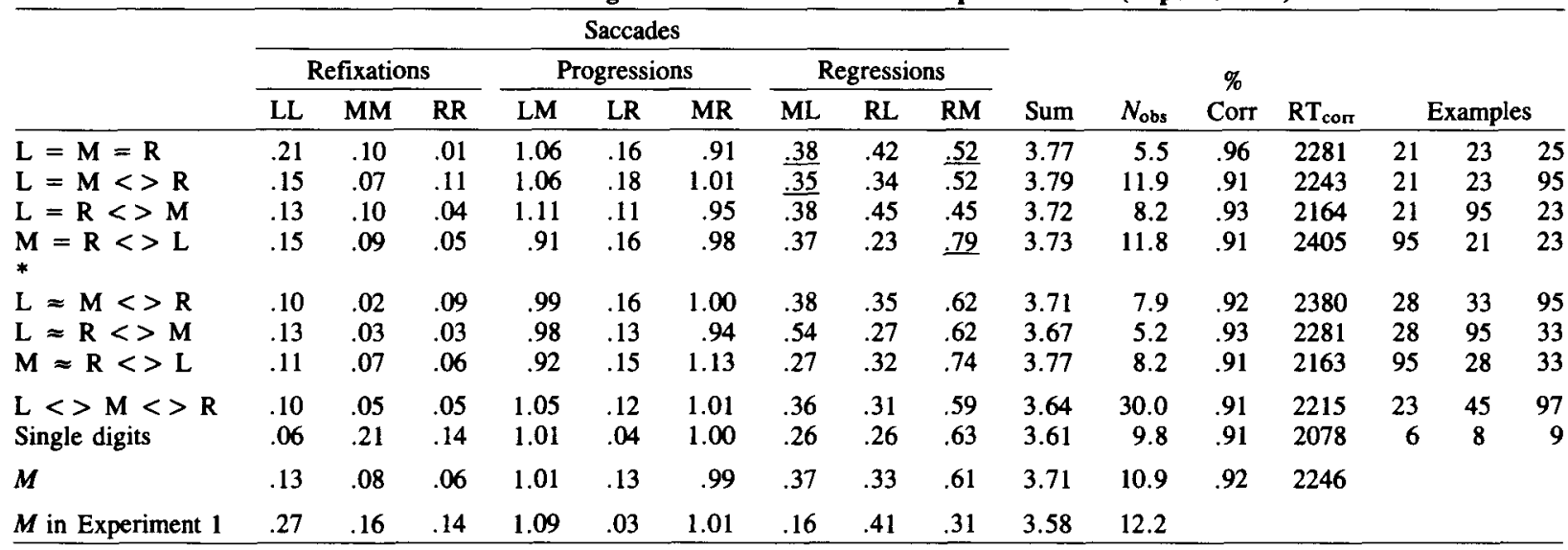

Note-L, M, and R = left, middle, and right numbers, respectively. " =" means same first digit. " $\approx$ " means first digits were different, but maximum difference in magnitude $\leq 10$. " $\langle>$ " means first digits were different and maximum difference in magnitude $>10$. $* \mathrm{~L} \approx \mathrm{M}$ $\approx \mathrm{R}$ was not included because there were only 0.5 observations on the average.

However, if we look at the regressions for numbers with a tie between their first digits (see data underlined in Table 3), we see no difference from regressions for other stimulus configurations. If the second and the first numbers start with the same digit, there are, on the average, . 37 regressions from the second to the first number, which is exactly the mean number of regressions for numbers that do not share the first digit. If the second and the third numbers start with the same digit, we have .52 regressions if all three numbers start with the same digit, and .79 regressions if only the second and the third numbers share the first digit. The figure of .79 is slightly above the mean value of .61 and therefore might give some support to the hypothesis of an increased need for regressions if numbers cannot be processed on the basis of the first digit alone. However, numbers that share the first digit are also closely related in magnitude. Therefore, the true control condition must consist of numbers that do not share the first number but are closely related in magnitude. An example would be "95 28 33," in comparison with "95 2123 ." These data can be found in the seventh line of Table 3. For this control subsample, the mean number of regressions from the third to the second number amounted to .74 , which is quite close to the .79 value found for numbers with the same first digit. Table 3 further shows that the lack of more regressions between numbers with the same digit did not result in higher error rates or very prolonged reaction times. Therefore, we think it can safely be concluded that the alternative strategy of only processing the first digits of numbers was not followed by the subjects of Experiment 2, and that the task dependence of the syllablelength effect in number processing is genuine.

For purposes of comparison, the mean number of saccades for Experiment 1 has also been included in the last line of Table 3 . The mean number of saccades amounted here to 3.58; two of these saccades were again progressions from the first to the second number, and from the second to the third number. For the rest, refixations tend to be higher and regressions lower. The former agrees with the larger FGD in Experiment 1 as opposed to Experiment 2.

\section{Discussion}

A nonrecall task in which numbers had to be compared was used to investigate whether the syllable-length effect would remain valid when vocalization was not required and numbers were presented on an ordinal scale. As in Experiment 1, possible confounding variables such as number frequency and number magnitude were controlled for by multiple regressions of the eye-fixation data based on all numbers smaller than 100 .

Neither FFD nor FGD was predicted by syllable length, but both had significant correlations with number magnitude (see Table 2 , but see note 2 ). The effect of number magnitude amounted to 0.25 msec per unit for FFD and $0.36 \mathrm{msec}$ for FGD. It is not clear what exactly caused the relationship between number magnitude and gaze duration, but an analogous finding has repeatedly been reported in number-comparison studies (see, e.g., Potts et al., 1978). Reaction time for deciding which digit of a pair is larger declines with the difference in magnitude between the digits, but it increases as the magnitude of the smaller digit increases. One explanation of the latter effect is that the internal representation of number magnitude is a nonlinear, compressed (e.g., logarithmic) function of the magnitude of the number. In such a compressive spacing, large numbers are closer together on the internal scale than small numbers, so that pairs at the lower end of the continuum are more discriminable than pairs at the upper end (see also Krueger, 1989, pp. 256-258). 
The greater difficulty in comparing large numbers may have resulted in longer gaze durations on large numbers than on small numbers, though additional experimental work is needed to confirm the hypothesis.

The failure to find a syllable-length effect in Experiment 2 runs against claims made by some authors (Rayner \& Pollatsek, 1989, p. 214; Rossi, 1985) about the generality of Pynte's (1974) results. No phonological code is needed in number processing when vocalization is not required and numbers have to be compared. Arguments that the experimental task did not provide the opportunity for the syllable-length effect to emerge because the numbers did not have to be fully processed are not supported by the empirical data. The pattern of eye movements (refixations, progressions, regressions) remained stable for different ties among the numbers of a stimulus display (see Table 3), which suggests that the numbers were indeed processed completely during the first gaze.

\section{GENERAL DISCUSSION}

Two experiments were run to investigate the relationship between syllable length of number names and eyefixation durations during silent reading of one- and twodigit numbers. In the first experiment, subjects had to read a series of three numbers and recall them orally; in the second experiment, subjects had to indicate manually whether the value of the middle number was between the values of the outer numbers. Special care was taken to make the control variables identical in both experiments (e.g., subjects from the same population; same equipment; same stimulus presentation; experiments run in the same 1-week period). Syllable length of number names accounted for $46 \%$ of the variance of first-gaze durations in Experiment 1, whereas it explained less than $1 \%$ of the variance in Experiment 2. This provides strong evidence for the claim that the phonological code of number names was assessed in the first experiment, but not in the second.

The syllable-length effect in number processing is regularly cited within the larger theoretical framework of the necessity of phonological recoding in visual processing (especially in word reading). Two opposing viewpoints prevail: Some authors claim that phonological recoding is an automatic and essential part of reading (e.g., Lukatela \& Turvey, 1990; Perfetti, Bell, \& Delaney, 1988; Rossi, 1985; Van Orden, Johnston, \& Hale, 1988), whereas others consider the phonological route helpful but not imperative (e.g., Brysbaert \& Praet, in press; Foster, 1990; Humphreys \& Evett, 1985; Klapp, Anderson, \& Berrian, 1973). At least for number processing, the latter view seems to be supported by the present study. Phonological recoding sometimes co-occurs with (silent) visual processing, but the activation (or at least the use) of the phonological code depends on specific task requirements. The fact that syllable length had an effect on first-fixation duration as well as on first-gaze duration in Experiment 1 suggests that the use of the phonological code occurs early in processing (Inhoff, 1984; Rayner \& Pollatsek, 1987).

Task-dependent syllable-length effects have been reported for other stimuli as well. Klapp, Anderson, and Berrian (1973), who used words and pictures, found that response latencies increased with syllables for naming but not for categorization, which made them conclude that the syllablelength effect was due to response preparation. Similarly, Brysbaert and d'Ydewalle (1991) reported a larger effect of word length in a word-naming task than in a lexical decision task (but see Balota \& Chumbley, 1984, and Hudson \& Bergman, 1985). ${ }^{5}$

Response preparation need not be the only reason for the syllable-length effect. In their general discussion, Klapp et al. (1973) mentioned rehearsal as a second mechanism that might be likely to elicit implicit speech in visual perception. They argued that

implicit speech may be used for memory of individual words, whether in a rote learning situation or in a situation where meaning is not easily assigned to the word strings so that the Ss choose to remember the words in a rote fashion.... Perhaps implicit as well as overt speech represents a method of processing to increase memory rather than a mode of processing which mediates comprehension. (p. 373)

So, according to Klapp et al. (1973), speech may have been involved in Experiment 1 simply to help memory performance in a rote learning situation, which is known to be supported by rehearsal. New experiments are planned to investigate which of the two explanations is the more plausible.

\section{REFERENCES}

Balota, D. A., Chumbley, J. I. (1984). Are lexical decisions a good measure of lexical access? The role of word frequency in the neglected decision stage. Journal of Experimental Psychology: Human Perception \& Performance, 10, 340-357.

BRYSBAERT, M. (1991). Algorithms for randomness in the behavioral sciences: A tutorial. Behavior Research Methods, Instruments, \& Computers, 23, 45-60.

BrysbaerT, M., D'YDEwAlle, G. (1991). A mathematical analysis of the convenient viewing position hypothesis and its components. In R. Schmid \& D. Zambarbieri (Eds.), Oculomotor control and cognitive processes: Normal and pathological aspects (pp. 331-340). Amsterdam: North-Holland.

Brysbaert, M., \& Praet, C. (in press). Reading isolated words: No evidence for automatic incorporation of the phonetic code. Psychological Research.

De Graef, P., Christiaens, D., D'Ydewalle, G. (1990). Perceptual effects of scene context on object identification. Psychological Research, 52, 317-329.

Eriksen, C. W., Pollack, M. D., \& Montague, W. E. (1970). Implicit speech: Mechanism in perceptual encoding? Journal of Experimental Psychology, 84, 502-507.

Foster, K. I. (1990). Lexical processing. In D. N. Osherson \& H. Lasnik (Eds.), An invitation to cognitive science: Vol. 1. Language (pp. 95-131). Cambridge, MA: MIT Press.

Galbrarth, R. C., \& Underwood, B. J. (1973). Perceived frequency of concrete and abstract words. Memory \& Cognition, 1, 56-60.

GernSBACHER, M. A. (1984). Resolving 20 years of inconsistent inter- 
actions between lexical familiarity and orthography, concreteness, and polysemy. Joumal of Experimental Psychology: General, 113, 256-281.

Hays, W. L. (1988). Statistics. New York: Holt, Rinehart \& Winston. Hudson, P. T., Bergman, M. W. (1985). Lexical knowledge in word recognition: Word length and word frequency in naming and lexical decision tasks. Joumal of Memory \& Language, 24, 46-58.

Humphreys, G. W., EveTt, L. J. (1985). Are there independent lexical and nonlexical routes in word processing? An evaluation of the dual-route theory of reading. Behavioral \& Brain Sciences, 8, 689-740.

HuNG, D. L., \& TzENG, O. J. (1981). Orthographic variations and visual information processing. Psychological Bulletin, 90, 377-414.

INHOFF, A. W. (1984). Two stages of word processing during eye fixations in the reading of prose. Journal of Verbal Learning \& Verbal Behavior, 23, 612-624.

KIRK, R. E. (1982). Experimental design: Procedures for the behavioral sciences. Monterey, CA: Brooks/Cole.

KLAPP, S. (1971). Implicit speech inferred from response latencies in same-different decisions. Journal of Experimental Psychology, 91, 262-267.

KLAPP, S. (1974). Syllable-dependent pronunciation latencies in number naming: A replication. Joumal of Experimental Psychology, 102, 1138-1140.

Klapp, S., Anderson, W., Berrin, R. (1973). Implicit speech in reading, reconsidered. Journal of Experimental Psychology, 100, 368-374.

KLIegl, R., Olson, R. K. (1981). Reduction and calibration of eye monitor data. Behavior Research Methods \& Instrumentation, 13, 107-111.

Krugger, L. E. (1989). Reconciling Fechner and Stevens: Toward a unified psychophysical law. Behavioral \& Brain Sciences, 12, 251-320.

LORCh, R. F., JR., a MYers, J. L. (1990). Regression analyses of repeated measures data in cognitive research. Journal of Experimental Psychology: Leaming, Memory, \& Cognition, 16, 149-157.

Lukatela, G., * Turvey, M. T. (1990). Automatic and pre-lexical computation of phonology in visual word identification. European Journal of Cognitive Psychology, 2, 325-343.

Perfetti, C. A., Bell, L. C., Delaney, S. M. (1988). Automatic (prelexical) phonetic activation in silent word reading: Evidence from backward masking. Journal of Memory \& Language, 27, 59-70.

Potts, G. R., Banks, W. P., Kosslyn, S. M., Moyer, R. S., Ruley, C. A., SMITH, K. H. (1978). Encoding and retrieval in comparative judgments. In N. J. Castellan, Jr., \& F. Restle (Eds.), Cognitive theory (Vol. 3, pp. 242-308). Hillsdale, NJ: Erlbaum.

PYNTE, J. (1974). Readiness for pronunciation during the reading process. Perception \& Psychophysics, 16, 110-112.

RAYNER, K., PollatseK, A. (1987). Eye movements in reading: $\mathbf{A}$ tutorial review. In M. Coltheart (Ed.), Attention and performance XII (pp. 327-362). London: Erlbaum.

Rayner, K., Pollatsek, A. (1989). The psychology of reading. Englewood Cliffs, NJ: Prentice-Hall.

Rossi, J. P. (1985). Les mécanismes de la lecture. Paris: Publication de la Sorbonne.

ShaPiro, B. J. (1969). The subjective estimation of relative word frequency. Journal of Verbal Learning \& Verbal Behavior, 8, 248-251.

STEVENS, S. S. (Ed.) (1951). Handbook of experimental psychology. New York: Wiley.

Van Orden, G. C., Johnston, J. C., \& Hale, B. L. (1988). Word identification in reading proceeds from spelling to sound to meaning. Journal of Experimental Psychology: Learning, Memory, \& Cognition, 14, 371-386.

YATES, F. (1933). The analysis of replicated experiments when field results are incomplete. Empire Journal of Experimental Agriculture, 1, 129-142.

Young, L. R., \& SHEENA, D. (1975). Survey of eye movement recording methods. Behavior Research Methods \& Instrumentation, 7, 397-429.

ZIPF, G. K. (1935). The psycho-biology of Language. Boston, MA: Houghton Mifflin.

ZIPF, G. K. (1949). Human behavior and the principle of least effort. Cambridge, MA: Addison-Wesley.

\section{NOTES}

1. In reaction time studies, it is common to analyze only the results of correct trials. However, in this experiment, an error in the oral recall could be due to factors other than the encoding of the middle number, the factor we are interested in. This might be the reason why there are no differences between correct and incorrect trials. The same is true for Experiment 2.

2. Exact $p$ values are difficult to calculate in this kind of experiment, because data were not obtained from independent observations. The significance levels given, however, do agree with both the Fisher $r$-to- $Z$ transformation for independent observations (Hays, 1988, pp. 590-593, 619-620) and the Lorch and Myers (1990) test of regression analyses of repeated measures data-except for the FGD data of Table 2, where the Fisher transformation suggests significance beyond the .05 level, whereas the Lorch and Myers test did not yield significance $(p>.10)$.

3. The subjective frequency of the number " 90 " in the Appendix is artificially inflated because the experiment was finished at the end of 1990 , and thus subjects had been regularly confronted with the number in the recent past. The same was not true for Pynte (1974).

4. The authors would like to thank Garvin Chastain for pointing them to this alternative explanation.

5. Syllable length and length in letters can be exchanged in this study, because both variables have an intercorrelation of .96 .

APPENDIX

Number Magnitude, Syllable Length, Number Frequency, and Eye-Fixation Data for Experiments 1 and 2

\begin{tabular}{|c|c|c|c|c|c|c|c|c|c|c|}
\hline \multirow[b]{2}{*}{ Mag } & \multirow[b]{2}{*}{ Syl } & \multirow[b]{2}{*}{ Freq } & \multicolumn{4}{|c|}{ Experiment 1} & \multicolumn{4}{|c|}{ Experiment 2} \\
\hline & & & FFD & $S D$ & FGD & $S D$ & FFD & $S D$ & FGD & $S D$ \\
\hline 0 & 1 & 430 & 241 & 123 & 365 & 218 & 229 & 95 & 249 & 77 \\
\hline 1 & 1 & 465 & 227 & 77 & 251 & 68 & 256 & 108 & 335 & 143 \\
\hline 2 & 1 & 435 & 221 & 61 & 231 & 53 & 205 & 58 & 243 & 126 \\
\hline 3 & 1 & 405 & 204 & 82 & 265 & 107 & 235 & 81 & 264 & 80 \\
\hline 4 & 1 & 375 & 208 & 81 & 310 & 186 & 212 & 70 & 234 & 76 \\
\hline 5 & 1 & 420 & 205 & 51 & 262 & 99 & 226 & 67 & 315 & 162 \\
\hline 6 & 1 & 365 & 193 & 88 & 243 & 99 & 230 & 94 & 249 & 86 \\
\hline 7 & 2 & 360 & 209 & 111 & 205 & 116 & 221 & 69 & 278 & 89 \\
\hline 8 & 1 & 355 & 209 & 74 & 262 & 48 & 235 & 116 & 285 & 105 \\
\hline 9 & 2 & 400 & 248 & 89 & 326 & 225 & 196 & 92 & 284 & 133 \\
\hline 10 & 1 & 445 & 275 & 146 & 312 & 141 & 188 & 109 & 240 & 124 \\
\hline$*_{11 L}$ & $1 \mathrm{~L}$ & $295 \mathrm{~L}$ & 227 & 76 & 353 & 238 & 199 & 64 & 250 & 47 \\
\hline 12 & 1 & 315 & 267 & 167 & 335 & 132 & 263 & 112 & 321 & 227 \\
\hline 13 & 2 & 350 & 308 & 114 & 349 & 118 & 253 & 106 & 273 & 96 \\
\hline
\end{tabular}


APPENDIX (Continued)

\begin{tabular}{|c|c|c|c|c|c|c|c|c|c|c|}
\hline \multirow[b]{2}{*}{ Mag } & \multirow[b]{2}{*}{ Syl } & \multirow[b]{2}{*}{ Freq } & \multicolumn{4}{|c|}{ Experiment 1} & \multicolumn{4}{|c|}{ Experiment 2} \\
\hline & & & FFD & $S D$ & FGD & $S D$ & FFD & $S D$ & FGD & $S D$ \\
\hline 14 & 2 & 320 & 218 & 122 & 304 & 168 & 245 & 147 & 420 & 166 \\
\hline 15 & 2 & 345 & 246 & 115 & 375 & 103 & 219 & 101 & 305 & 62 \\
\hline 16 & 2 & 325 & 316 & 160 & 373 & 146 & 290 & 85 & 309 & 89 \\
\hline 17 & 3 & 290 & 240 & 101 & 306 & 107 & 226 & 73 & 254 & 67 \\
\hline 18 & 2 & 365 & 214 & 143 & 320 & 146 & 305 & 208 & 387 & 242 \\
\hline 19 & 3 & 275 & 258 & 163 & 331 & 142 & 221 & 89 & 333 & 96 \\
\hline$* 20 \mathrm{~L}$ & $2 \mathrm{~L}$ & $370 \mathrm{H}$ & 255 & 118 & 297 & 97 & 200 & 87 & 326 & 115 \\
\hline$* 21 \mathrm{~L}$ & $4 \mathrm{H}$ & $340 \mathrm{H}$ & 212 & 126 & 343 & 119 & 245 & 161 & 295 & 162 \\
\hline 22 & 4 & 260 & 357 & 242 & 382 & 279 & 223 & 66 & 233 & 70 \\
\hline 23 & 4 & 200 & 272 & 158 & 403 & 146 & 205 & 94 & 245 & 96 \\
\hline 24 & 4 & 265 & 270 & 129 & 434 & 138 & 187 & 56 & 286 & 105 \\
\hline$* 25 \mathrm{~L}$ & $4 \mathrm{H}$ & $370 \mathrm{H}$ & 284 & 131 & 403 & 133 & 207 & 100 & 325 & 164 \\
\hline 26 & 4 & 245 & 285 & 105 & 425 & 161 & 225 & 97 & 375 & 231 \\
\hline 27 & 5 & 240 & 334 & 200 & 482 & 151 & 215 & 90 & 257 & 95 \\
\hline$* 28 \mathrm{~L}$ & $4 \mathrm{H}$ & $225 \mathrm{~L}$ & 291 & 212 & 459 & 182 & 244 & 73 & 282 & 96 \\
\hline 29 & 5 & 200 & 214 & 144 & 381 & 151 & 253 & 131 & 324 & 102 \\
\hline *30L & $2 \mathrm{~L}$ & $320 \mathrm{H}$ & 174 & 89 & 306 & 135 & 204 & 71 & 292 & 110 \\
\hline$* 31 \mathrm{~L}$ & $4 \mathrm{~L}$ & $140 \mathrm{~L}$ & 304 & 135 & 390 & 153 & 258 & 100 & 282 & 91 \\
\hline 32 & 4 & 260 & 252 & 138 & 449 & 170 & 220 & 106 & 305 & 117 \\
\hline 33 & 4 & 215 & 206 & 105 & 395 & 98 & 238 & 153 & 345 & 124 \\
\hline *34 L & $4 \mathrm{H}$ & $205 \mathrm{~L}$ & 242 & 130 & 384 & 215 & 241 & 104 & 278 & 93 \\
\hline 35 & 4 & 240 & 233 & 131 & 357 & 155 & 210 & 85 & 305 & 176 \\
\hline 36 & 4 & 260 & 281 & 188 & 407 & 163 & 195 & 134 & 298 & 131 \\
\hline 37 & 5 & 175 & 424 & 244 & 527 & 235 & 191 & 78 & 223 & 83 \\
\hline 38 & 4 & 195 & 268 & 180 & 507 & 302 & 225 & 123 & 298 & 134 \\
\hline 39 & 5 & 190 & 316 & 172 & 447 & 182 & 343 & 252 & 452 & 237 \\
\hline 40 & 2 & 300 & 276 & 111 & 337 & 102 & 238 & 114 & 275 & 117 \\
\hline 41 & 4 & 175 & 217 & 92 & 359 & 124 & 225 & 86 & 238 & 82 \\
\hline 42 & 4 & 220 & 235 & 149 & 385 & 155 & 245 & 125 & 287 & 100 \\
\hline 43 & 4 & 130 & 273 & 134 & 435 & 258 & 215 & 102 & 248 & 100 \\
\hline 44 & 4 & 165 & 305 & 153 & 374 & 180 & 245 & 81 & 276 & 124 \\
\hline 45 & 4 & 270 & 323 & 204 & 493 & 166 & 236 & 104 & 313 & 67 \\
\hline 46 & 4 & 220 & 292 & 182 & 436 & 152 & 198 & 94 & 271 & 127 \\
\hline 47 & 5 & 230 & 305 & 176 & 448 & 137 & 275 & 176 & 375 & 181 \\
\hline 48 & 4 & 175 & 245 & 123 & 547 & 169 & 203 & 76 & 319 & 173 \\
\hline 49 & 5 & 175 & 334 & 187 & 608 & 210 & 209 & 95 & 288 & 102 \\
\hline$* 50 \mathrm{H}$ & $2 \mathrm{~L}$ & $330 \mathrm{H}$ & 266 & 115 & 324 & 96 & 245 & 122 & 280 & 124 \\
\hline 51 & 4 & 160 & 303 & 117 & 424 & 217 & 231 & 140 & 288 & 156 \\
\hline 52 & 4 & 140 & 313 & 198 & 405 & 182 & 211 & 85 & 241 & 89 \\
\hline 53 & 4 & 125 & 338 & 191 & 458 & 195 & 237 & 137 & 288 & 117 \\
\hline 54 & 4 & 195 & 302 & 221 & 470 & 211 & 233 & 129 & 375 & 271 \\
\hline 55 & 4 & 235 & 269 & 112 & 437 & 246 & 229 & 67 & 289 & 107 \\
\hline 56 & 4 & 185 & 241 & 84 & 411 & 170 & 233 & 114 & 292 & 177 \\
\hline 57 & 5 & 165 & 272 & 189 & 572 & 395 & 206 & 121 & 275 & 181 \\
\hline 58 & 4 & 170 & 299 & 184 & 454 & 163 & 246 & 136 & 338 & 100 \\
\hline 59 & 5 & 150 & 345 & 287 & 660 & 541 & 237 & 126 & 337 & 221 \\
\hline$* 60 \mathrm{H}$ & $2 \mathrm{~L}$ & $290 \mathrm{~L}$ & 230 & 94 & 336 & 183 & 200 & 84 & 264 & 87 \\
\hline 61 & 4 & 165 & 261 & 101 & 333 & 93 & 212 & 80 & 272 & 110 \\
\hline$* 62 \mathrm{H}$ & $4 \mathrm{H}$ & $165 \mathrm{~L}$ & 215 & 152 & 425 & 183 & 215 & 106 & 364 & 119 \\
\hline 63 & 4 & 160 & 255 & 92 & 384 & 128 & 228 & 77 & 290 & 135 \\
\hline 64 & 4 & 195 & 292 & 163 & 407 & 176 & 238 & 81 & 330 & 172 \\
\hline 65 & 4 & 200 & 269 & 164 & 398 & 162 & 265 & 116 & 344 & 191 \\
\hline 66 & 4 & 215 & 202 & 94 & 343 & 155 & 192 & 67 & 256 & 67 \\
\hline 67 & 5 & 195 & 316 & 232 & 503 & 220 & 352 & 322 & 420 & 302 \\
\hline 68 & 4 & 235 & 301 & 198 & 520 & 190 & 206 & 103 & 349 & 182 \\
\hline$* 69 \mathrm{H}$ & $5 \mathrm{H}$ & $325 \mathrm{H}$ & 280 & 136 & 402 & 189 & 307 & 153 & 420 & 186 \\
\hline 70 & 3 & 245 & 278 & 112 & 372 & 123 & 229 & 149 & 322 & 166 \\
\hline 71 & 5 & 240 & 262 & 127 & 332 & 135 & 300 & 218 & 348 & 215 \\
\hline 72 & 5 & 220 & 225 & 98 & 396 & 146 & 275 & 123 & 298 & 121 \\
\hline *73 H & $5 \mathrm{H}$ & $145 \mathrm{~L}$ & 349 & 192 & 466 & 122 & 208 & 117 & 243 & 100 \\
\hline
\end{tabular}


APPENDIX (Continued)

\begin{tabular}{|c|c|c|c|c|c|c|c|c|c|c|}
\hline \multirow[b]{2}{*}{ Mag } & \multirow[b]{2}{*}{ Syl } & \multirow[b]{2}{*}{ Freq } & \multicolumn{4}{|c|}{ Experiment 1} & \multicolumn{4}{|c|}{ Experiment 2} \\
\hline & & & FFD & $S D$ & FGD & $S D$ & FFD & $S D$ & FGD & $S D$ \\
\hline 74 & 5 & 200 & 235 & 122 & 495 & 155 & 211 & 99 & 330 & 240 \\
\hline$* 75 \mathrm{H}$ & $5 \mathrm{H}$ & $360 \mathrm{H}$ & 360 & 182 & 474 & 189 & 226 & 82 & 317 & 114 \\
\hline 76 & 5 & 190 & 261 & 159 & 535 & 246 & 204 & 93 & 300 & 148 \\
\hline 77 & 6 & 200 & 241 & 107 & 325 & 126 & 296 & 261 & 296 & 261 \\
\hline 78 & 5 & 140 & 386 & 224 & 579 & 159 & 228 & 84 & 274 & 93 \\
\hline 79 & 6 & 155 & 240 & 138 & 417 & 150 & 228 & 149 & 305 & 152 \\
\hline$* 80 \mathrm{H}$ & $2 \mathrm{~L}$ & $260 \mathrm{~L}$ & 203 & 76 & 318 & 101 & 269 & 143 & 351 & 149 \\
\hline 81 & 4 & 205 & 253 & 171 & 358 & 174 & 238 & 92 & 316 & 119 \\
\hline 82 & 4 & 170 & 271 & 125 & 359 & 137 & 238 & 107 & 300 & 89 \\
\hline 83 & 4 & 145 & 244 & 132 & 469 & 141 & 258 & 114 & 314 & 103 \\
\hline 84 & 4 & 215 & 282 & 163 & 460 & 105 & 265 & 95 & 293 & 94 \\
\hline 85 & 4 & 200 & 280 & 178 & 486 & 168 & 206 & 90 & 283 & 117 \\
\hline 86 & 4 & 150 & 253 & 97 & 506 & 179 & 205 & 78 & 303 & 128 \\
\hline 87 & 5 & 145 & 295 & 227 & 497 & 260 & 268 & 162 & 313 & 138 \\
\hline 88 & 4 & 205 & 236 & 102 & 391 & 171 & 260 & 121 & 297 & 113 \\
\hline 89 & 5 & 235 & 281 & 173 & 455 & 137 & 244 & 93 & 320 & 160 \\
\hline$* 90 \mathrm{H}$ & $3 \mathrm{~L}$ & $360 \mathrm{H}$ & 256 & 164 & 359 & 155 & 255 & 83 & 321 & 103 \\
\hline 91 & 5 & 235 & 246 & 139 & 425 & 216 & 240 & 97 & 279 & 98 \\
\hline 92 & 5 & 245 & 213 & 144 & 359 & 164 & 295 & 105 & 305 & 120 \\
\hline 93 & 5 & 150 & 253 & 114 & 439 & 177 & 202 & 101 & 337 & 219 \\
\hline 94 & 5 & 165 & 283 & 169 & 519 & 249 & 321 & 135 & 365 & 123 \\
\hline 95 & 5 & 245 & 223 & 116 & 481 & 224 & 225 & 158 & 302 & 179 \\
\hline 96 & 5 & 200 & 305 & 212 & 490 & 193 & 273 & 148 & 316 & 122 \\
\hline 97 & 6 & 160 & 243 & 123 & 461 & 203 & 278 & 117 & 345 & 124 \\
\hline 98 & 5 & 205 & 317 & 179 & 495 & 153 & 215 & 58 & 308 & 98 \\
\hline 99 & 6 & 280 & 235 & 115 & 312 & 129 & 215 & 79 & 310 & 92 \\
\hline
\end{tabular}

Note-FFD = first-fixation duration. FGD = first-gaze duration. ${ }^{*}$ Indicates a number of the number subset on which the ANOVA was conducted; $\mathrm{L}$ and $\mathrm{H}$ stand, respectively, for low and high value for the predictor variables number magnitude (Mag), syllable length of the number name (Syl), and number frequency (Freq).

(Manuscript received January 17, 1991;

revision accepted for publication July $15,1991$. 